\title{
The macroscopic yield behaviour of polymers
}

\author{
RAM RAGHAVA, ROBERT M. CADDELL \\ Department of Mechanical Engineering, The University of Michigan, Ann Arbor, Michigan, USA \\ GREGORY S. Y. YEH \\ Department of Materia/s and Metallurgical Engineering, The University of Michigan, \\ Ann Arbor, Michigan, USA
}

\begin{abstract}
A yield criterion, not previously compared with the actual macroscopic behaviour of polymers, is herein compared with the pressure-modified octahedral shear stress criterion earlier suggested by others. This new relation, which is a version of the von Mises criterion, accommodates differences in tensile and compressive yield strengths and accounts for any dependence of yielding on the hydrostatic component of the applied stress state.

With the use of thin-wall tubes accounting for the majority of experimental points, the yield behaviour of polycarbonate and polyvinylchloride was investigated. Besides these findings, results previously reported by others have also been utilized in this paper. Since these various studies employed quite different polymers, the excellent overall correlation of experiment with prediction should merit the serious attention of persons interested in the macroscopic yield behaviour of polymers.

Comparisons between this new criterion and the modified octahedral shear stress are also made in regard to the effect of pressure on subsequent yield behaviour. Although not fully verified, it is suggested that the predictions which result using this new criterion, seem a little more reasonable.
\end{abstract}

\section{Introduction}

A recent paper by Ward [1] contained both a review of yield criteria and a survey of work involving the yield behaviour of polymers. Classical plasticity theory often includes several assumptions where yield behaviour of solids is involved; these are: (1) the material is isotropic and homogeneous; (2) yielding is uninfluenced by the hydrostatic component of the stress state; (3) tensile and compressive yield strengths are equal; (4) deformation proceeds under constant volume. Although these assumptions are reasonable where ductile metals are involved, their accuracy diminishes when polymers are the solids under consideration.

Yield criteria (also called "failure theories") have existed for over a century but only within the last decade or so has serious effort been expended to test these criteria against the yield behaviour of various polymers. Some of the more widely quoted publications [2-4] have shown reasonable correlation between predicted (C) 1973 Chapman and Hall Ltd. and measured values of yielding for, perhaps, one specific polymer. As mentioned recently by Brown [5] however, a generally accepted yield criterion for this class of solids is still lacking.

Evidence to date indicates that a sensible criterion must at the very least include pressure dependency effects on yielding and, in many cases, the difference in tensile and compressive yield strengths must be accommodated. Certainly the added effects of strain-rate, temperature, and anisotropy must be studied eventually but the complexities they will introduce are not at all considered further in this paper.

Recently, the predictions based upon a modified von Mises yield criterion have been compared with experimental results obtained in our laboratories; the correlation seems excellent for the two different polymers employed as test specimens. Additionally, results with other polymers as reported by others [2-4] have also correlated quite well with this criterion.

It must be noted that Meldahl [6] presented 225 
this criterion in the form of a three-dimensional model of a yield surface; he attributed the concept to Schleicher [7]. More recently, Stassi D'Alia [8] has suggested this as a universal yield criterion while Tschoegl [9], who seems unaware of Stassi D'Alia's work, has considered this as one of several possible "failure" surfaces. To our knowledge, no one to date has tested this concept against experimental findings using polymeric solids; in view of the agreement found it seems appropriate to suggest that future studies of yielding should subject this criterion to at least a reasonable degree of scrutiny.

\section{Analytical background}

In its most general form, the proposed criterion for macroscopic yielding may be expressed as:

$$
\begin{gathered}
\left(\sigma_{1}-\sigma_{2}\right)^{2}+\left(\sigma_{2}-\sigma_{3}\right)^{2}+\left(\sigma_{3}-\sigma_{1}\right)^{2}+ \\
2(C-T)\left(\sigma_{1}+\sigma_{2}+\sigma_{3}\right)=2 C T
\end{gathered}
$$

where $\sigma_{1}, \sigma_{2}$, and $\sigma_{3}$ are, as in the usual notation, the three principal stresses of the applied stress state while $C$ and $T$ represent the absolute values of the compressive and tensile yield strengths respectively. Note that for this entire paper, the use of $C$ and $T$ always pertains to absolute values. If $C=T$ then Equation 1 reduces to the usual form of the von Mises criterion. The influence of the hydrostatic portion of the applied stress state is introduced by the quantity $\left(\sigma_{1}+\sigma_{2}+\sigma_{3}\right)$ which is the first invariant of the stress tensor or, equivalently, three times the "mean" stress.

For experimental simplicity, yield studies are most easily conducted using uniaxial and biaxial types of loading situations. Consequently, for the purposes of this present study, Equation 1 can be rewritten for the case where $\sigma_{3}=0$ to give:

$\sigma_{1}^{2}+\sigma_{2}^{2}-\sigma_{1} \sigma_{2}+(C-T)\left(\sigma_{1}+\sigma_{2}\right)=C T$.

By defining two normalizing factors, $R_{1}=\sigma_{1} / T$ and $R_{2}=\sigma_{2} / T$ there then results:

$$
\begin{aligned}
R_{1}{ }^{2}+R_{2}{ }^{2}-R_{1} R_{2}+ & \left(\frac{C}{T}-1\right) \\
& \left(R_{1}+R_{2}\right)=C / T .
\end{aligned}
$$

Because of detailed comparisons that will be made, we introduce the form of the yield criterion suggested by Bauwens [3] and Sternstein [4] which had been initially proposed by Nadai (e.g. [10]). Although recently [11] there has been posed an argument which states that the physical basis leading to this criterion differs between [3] and [4], the fact remains that the form of the criterion itself is really identical.

In general form this criterion is:

$$
\tau_{0}+A \sigma_{\mathrm{m}}=\text { constant } .
$$

Here, $\tau_{0}$ is the octahedral shear stress, $\sigma_{\mathrm{m}}$ is the hydrostatic or mean stress, and $A$ is a constant. Note that although Equation 4 is another form of a pressure modified von Mises criterion it is not equivalent to Equation 1. For future comparative purposes with Equation 3, the normalized form of Equation 4 (with $\sigma_{3}=0$ ) can be expressed as:

$$
\begin{gathered}
\left(R_{1}^{2}+R_{2}^{2}-R_{1} R_{2}\right)^{\frac{1}{2}}+\frac{\left(R_{1}+R_{2}\right)\left(\frac{C}{T}-1\right)}{\left(\frac{C}{T}+1\right)} \\
=\frac{2 C / T}{\left(\frac{C}{T}+1\right)}
\end{gathered}
$$

\section{Experimental studies}

Two commercially supplied materials in the form of solid rods of $1.5 \mathrm{in}$. $(38 \mathrm{~mm})$ diameter were used in this study; they were polyvinylchloride (PVC) and polycarbonate (PC). All tests were conducted at room temperature (about $25^{\circ} \mathrm{C}$ ) using an Instron testing machine whose crosshead speed was $0.05 \mathrm{~cm} / \mathrm{min}$.

\subsection{Uniaxial tensile tests}

From the original rods, standard tensile specimens were made. Their overall length was $150 \mathrm{~mm}$ while the gauge section was $100 \mathrm{~mm}$ long and about $9 \mathrm{~mm}$ in diameter. The ends were threaded for adaptation to the machine grips and an Instron extensometer (type G-51-12M) was fitted to the test section; this provided drive to the recorder which led to a load-extension curve for each such test. Throughout the entire study, calibration checks of load and extensometer movement were made at particular intervals to ensure accuracy.

Each load-extension curve was reduced to a true stress-true strain plot; from these, yield strengths were determined using a strain displacement that was equivalent to a $0.3 \%$ offset in the usual terminology. Fig. 1 shows a typical test result where the yield strength for a $0.3 \%$ offset is indicated.

\subsection{Direct compression tests}

Right circular cylinders were made from the 


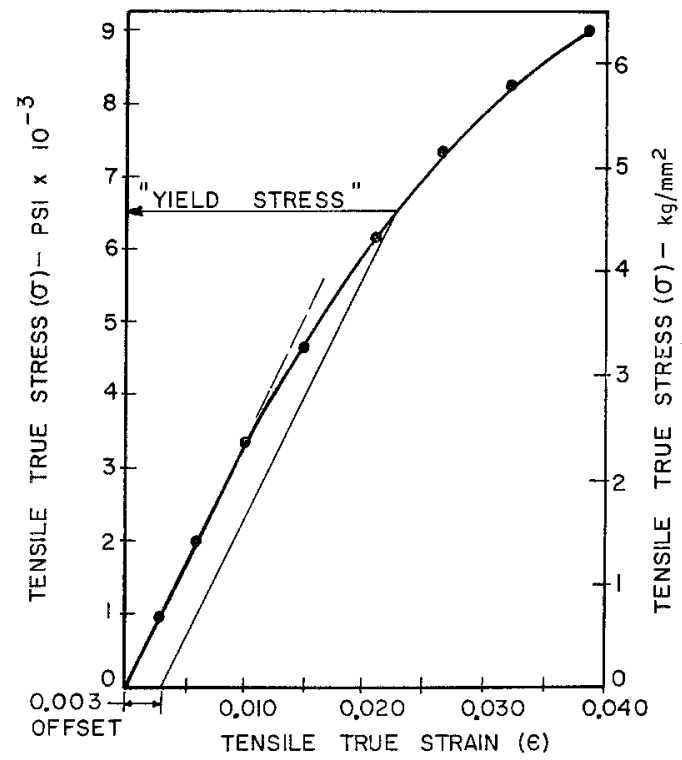

Figure $I$ Tensile true stress-true strain plot for PC illustrating the selection of "yield strength" as based upon an offset of $0.3 \%$ (i.e. a strain of 0.003 ).

original rod; to check possible directional effects, test specimens were machined from both the radial and axial directions of the large rod. All such specimens were about $19 \mathrm{~mm}$ high and $12 \times 12 \mathrm{~mm}$ cross section. Hardened and ground platens were used to transmit load and the platen-specimen interface was lubricated with a thin teflon sheet and molydisulphide paste. This was done to reduce the frictional effects that are encountered in such a test. During the loading sequence, readings of instantaneous height, cross-section, and load were determined. From these data, the desired true stress-strain values were found. The use of a $0.3 \%$ offset on the compressive true stress-strain plots, as in Fig. 1, again provided the yield strength as desired.

\subsection{Thin-wall tube tests}

Tubes were produced from the large rods to the general configuration shown in Fig. 2; those are PVC specimens that had been subjected to two different loading paths (i.e. constant ratios of $\left.\sigma_{1} / \sigma_{2}\right)$. The upper tube experienced internal pressure and axial compression whereas the lower tube was subjected to internal pressure and axial tension. By varying the stress ratio through this type of combined loading, a number of points were obtained for comparison with a yield locus based upon analytical predictions.

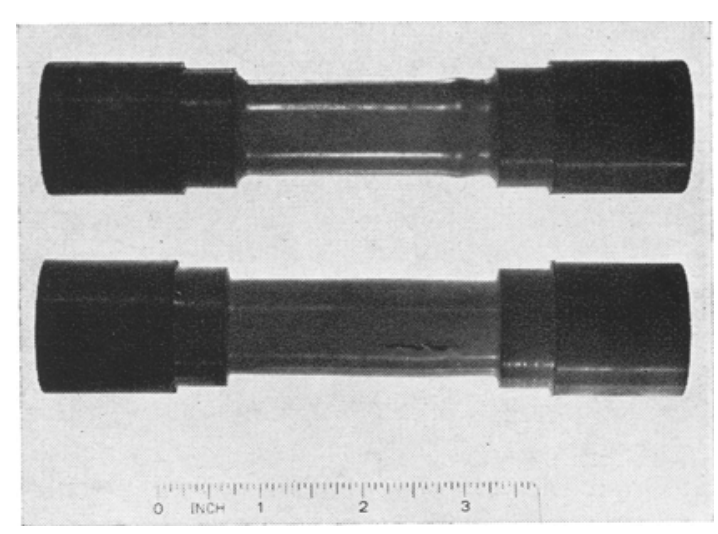

Figure 2 Typical tubular specimens of PVC. Top specimen was subjected to combined internal pressure and axial compression. Bottom specimen was subjected to combined internal pressure and axial tension.

Axial loads, whether tensile or compressive, were transmitted from the Instron loading head to the tube walls by means of special end adapters. These were fastened to the tube ends and their design was such that the tube shape was the same regardless of the type of load to be applied. Internal pressure was produced by a hand pump using a standard hydraulic oil. Because of the very slow axial loading rate it was possible to adjust the internal pressure such that the ratio of axial to hoop or circumferential stress (i.e. $\sigma_{1} / \sigma_{2}$ ) was for all practical purposes

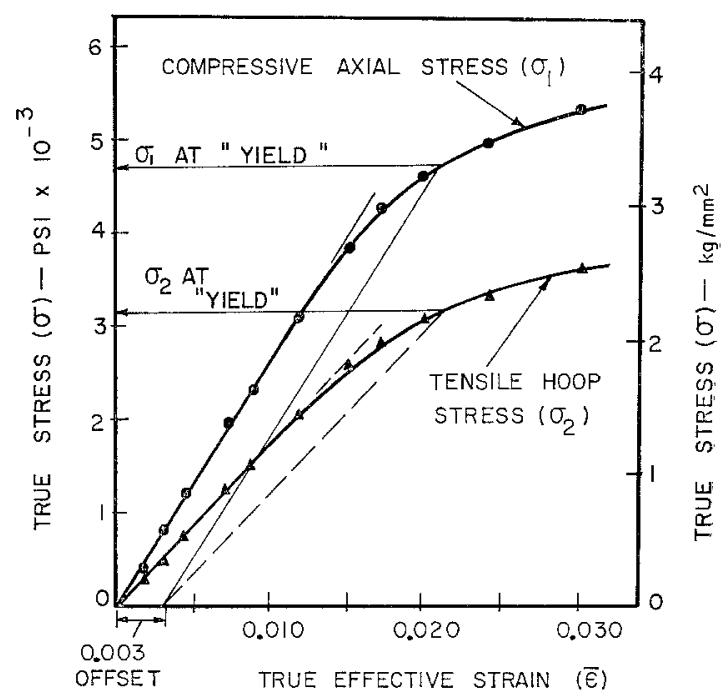

Figure 3 Biaxial stresses $\left(\sigma_{1}\right.$ and $\sigma_{2}$ ) versus effective strain ( $\epsilon$ ) using a PVC tube and a stress ratio $\left(\sigma_{1} / \sigma_{2}\right)$ of -1.5 (internal pressure and axial compression). Note the choice of stress at yield based upon a $0.3 \%$ offset. 
maintained at the constant value desired for a particular test. In truth, rather than this describing a linear loading path, it consisted of a number of small but discrete jumps whose average values were always within close agreement with the stress ratio desired. That this is so can be seen from Fig. 3 which shows the plots of hoop and axial stresses versus effective strain; the small deviations from a smooth line attest to the reasonableness of considering this test being conducted at a "constant" stress ratio. Two points are worth discussing here. First, to determine the values of $\sigma_{1}$ and $\sigma_{2}$ at which yielding occurs in a biaxial test, one must plot stresses versus strains before an offset method can be applied. For all of the biaxial tests conducted in this work, we have used the concept of "effective strain" in order to construct the necessary plots. This strain (for plane stress conditions as in these tube studies) is defined as:

$$
\begin{gathered}
\epsilon=\frac{1}{1+\mu}\left[\epsilon_{1}^{2}+\epsilon_{2}^{2}-\epsilon_{1} \epsilon_{2}+\right. \\
\left.\frac{\mu}{(1-\mu)^{2}}\left(\epsilon_{1}+\epsilon_{2}\right)^{2}\right)^{\frac{1}{2}}
\end{gathered}
$$

where $\mu$ is Poisson's ratio, determined to be 0.42 for $\mathrm{PC}$ and 0.38 for PVC in this study by measuring three principal strains simultaneously during tensile loading. Here $\epsilon_{1}$ and $\epsilon_{2}$ correspond to the strains in directions that define $\sigma_{1}$ and $\sigma_{2}$.

The stresses were found using:

$$
\begin{gathered}
\sigma_{1}=\frac{P d^{2}+4 L / \pi}{4 t(d+t)} \text { (axial stress) } \\
\sigma_{2}=\frac{P d}{2 t} \text { (hoop stress) }
\end{gathered}
$$

where $P, L, d$, and $t$ refer to internal pressure, axial load, tube diameter, and tube wall thickness respectively; a group of four such instantaneous measurements led to corresponding values of $\bar{\epsilon}, \sigma_{1}$, and $\sigma_{2}$ using Equations 6 to 8 . Details leading to Equations 7 and 8 may be found in [12]. Note that for $\mu$ equals $1 / 2$, Equation 6 becomes equivalent to:

$$
\bar{\epsilon}=\left[4 / 3\left(\epsilon_{1}^{2}+\epsilon_{2}^{2}+\epsilon_{3}^{2}\right)^{\frac{1}{2}}\right.
$$

which is the effective strain function for the von Mises criterion under the conditions of plane stress and constancy of volume. Note also that the axial stress is always referred to as $\sigma_{1}$ while the hoop stress is $\sigma_{2}$ in this paper. By determining a number of instantaneous load, elongation, diameter values, these data could then be reduced to provide the type of plot illustrated by Fig. 3 .
The second point of concern lies in the determination of the "values" of $\sigma_{1}$ and $\sigma_{2}$ which define yielding for a particular loading line. This was checked in two ways. If the $0.3 \%$ offset is applied to the $\sigma_{1}-\bar{\epsilon}$ curve, then the value of $\sigma_{1}$ at "yielding" is found. Since the stress ratio was constant for a given test, this was then used to define the corresponding value of $\sigma_{2}$. Alternately, the offset could be applied to the $\sigma_{2}-\bar{\epsilon}$ curve; this provided the value of $\sigma_{2}$ at yielding and $\sigma_{1}$ could then be computed as just indicated. It was found that either approach produced almost equivalent values of $\sigma_{1}$ and $\sigma_{2}$. Because there was a little less scatter of points for the higher level stress curve, preference was given to that curve using the offset and the corresponding lower stress at yielding was then found using the stress ratio. Both choices are illustrated in Fig. 3.

All tubes had a wall thickness of $1 \mathrm{~mm}$, a mean radius of $10.5 \mathrm{~mm}$, and a length of reduced gauge section of about $56 \mathrm{~mm}$. For each of these tests, the Instron chart was always driven by the output of an extensometer adapted to the gauge section; where axial loads were compressive, the extensometer was initially extended then allowed to compress during a test. The design of these devices permits this to be done quite easily.

\section{Comment on macroscopic isotropy}

From the compressive tests described under Section 3.2 it was observed that the "yield strength" in the radial direction of the test rod was equivalent to that observed using specimens produced from the axial direction. No pure compression tests were conducted on tubes because of problems with buckling.

In regard to tensile tests, those discussed under Section 3.1 as well as uniaxial tensile test using tubes without internal pressure were performed. These tests provided tensile yield data in the axial direction of the test rods; because of the limited size it was not possible to conduct tensile tests in the radial direction of the rod with the equipment at our disposal. However, tests were conducted to determine the tensile yield strength in the "hoop" or circumferential direction. This was done using what amounted to "open-ended" tubes such that the internal pressure did not induce any meaningful longitudinal stress in the tube wall, rather only a hoop stress was imposed. These results were practically equivalent to the other tensile results.

It is also noted that during the standard tensile 
tests, measurements of lateral dimensional changes in the gauge section were made at different orientations of the gauge diameter. Such measurements during a test showed equivalence at any instant.

From the above measurements it was concluded that for the purposes of this study, both polymers possessed macroscopic isotropy.

\section{Comparison of experimental results with analytical predictions}

As defined in Equation 3, the $C / T$ ratio was found to be 1.2 for $\mathrm{PC}$ and 1.33 for PVC. For reasons that will be explained shortly, a yield locus was determined for a ratio of 1.3 using Equation 3. This is the solid line in the form of an ellipse shown in Fig. 4. A number of points obtained from our experiments are plotted on this figure. In addition, data abstracted from other sources [2-4] are also included on this plot. Each of these other investigators found that for the particular polymer they used, the $C / T$ ratio was just about 1.3. It was for this reason that value was chosen to produce the plot on Fig. 4 . Note that as this ratio decreases towards unity the ellipse tends to move upward and to the right (along a line at $45^{\circ}$ ) thus the fit of the PC data would be even better if the proper value for that material (i.e. 1.2) had been used. In point of fact it did not seem necessary to repeat the drawing of Fig. 4 for several values of $C / T$ that differ so slightly.

With the experimental procedures used in our study it was not possible to produce second and third quadrant points; only those reported by Whitney [2] are shown, as the authors are unaware of any other such information. It should be pointed out that to use data reported by other workers, it is essential to have both the tensile and compressive yield strengths. As these are not reported in most other sources, we have, unfortunately, been forced to forego additional comparisons.

\section{Discussion on the comparison of yield criteria}

Using Equations 3 and 5 it is instructive to compare predicted yield surfaces for various $C / T$ ratios using each of these equations. For the sake of clarity, the ellipse of Fig. 4 is redrawn on Fig. 5 and a number of points which were calculated from Equation 5 have been added to this figure as solid circles. These points were based on a ratio of 1.3 also. At first glance, the

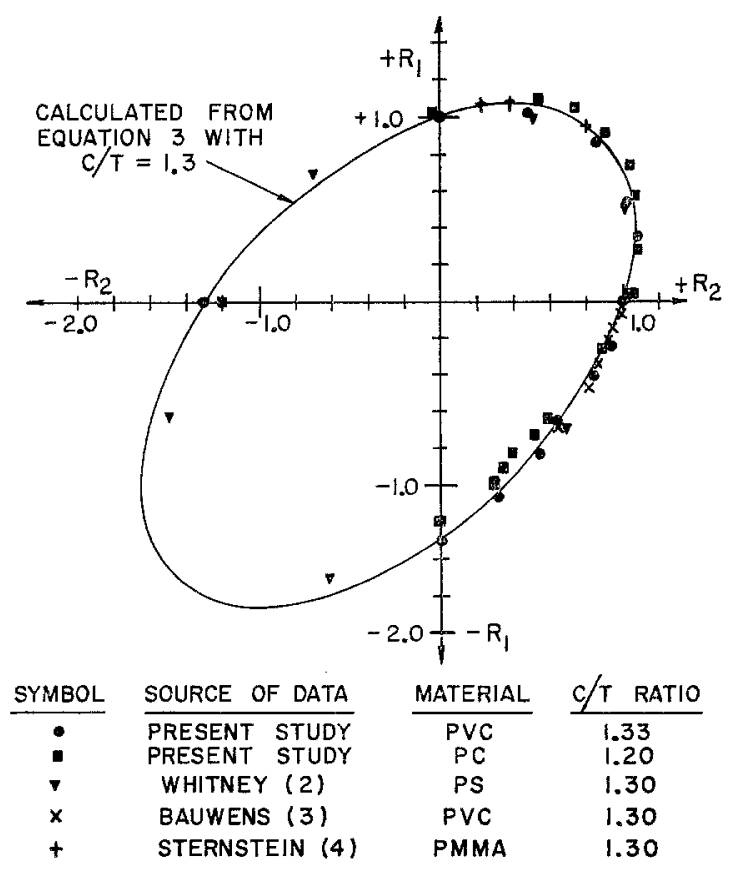

Figure 4 Comparison of predicted yield locus based upon Equation 3 for a $C / T$ ratio of 1.3 with experimental results from various sources as noted.

differences seem trivial but as the $C / T$ ratio increases, a much greater difference is found. This is especially true in the third quadrant where pressure effects are most pronounced. Fig. 6 duplicates Fig. 5 except the $C / T$ ratio is 2.0 . As discussed by Tschoegl [9], first quadrant points are quite insensitive in regard to describing a most appropriate "failure" surface, in fact, to determine with reasonable certainty which of these two criteria are more accurate, tests that lead to third quadrant points are essential. Where $C / T$ ratios are not much greater than unity and where third quadrant loading is absent, these criteria predict nearly equivalent results.

From another point of view, a further comparison between the two criteria can be made. Results such as reported by Ainbinder et al [13], Christiansen et al [14], and Sardar et al [15] indicate that the tensile and compressive yield strengths increase under increasing hydrostatic pressure. Consider that a hydrostatic pressure, $P$, is applied to a specimen which is then subjected to a tensile test. The stress state may be depicted as:

$$
\sigma_{2}=\sigma_{3}=-P, \quad \sigma_{1}=\sigma-P
$$




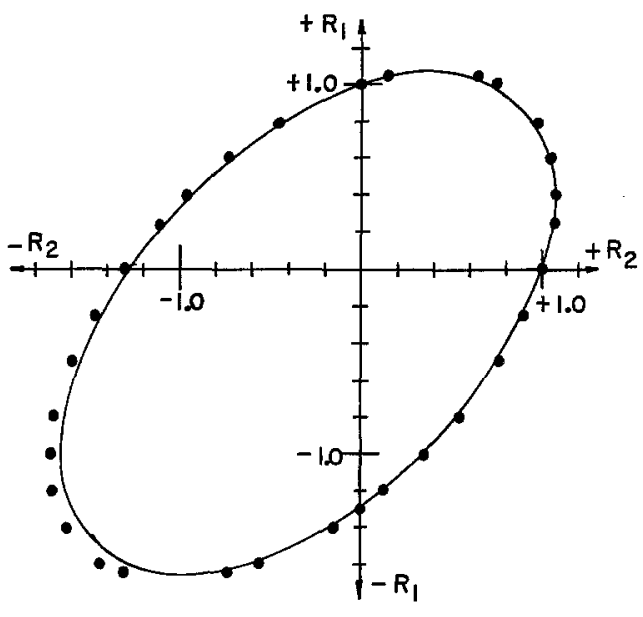

SOLID LINE (ELLIPSE) DETERMINED FROM EQUATION 3 WITH $C / T=1.3$

\section{- calculated from equation 5 with $\mathrm{C} / \mathrm{T}=1.3$}

Figure 5 Comparison of results predicted by Equations 3 and 5 for a $C / T$ ratio of 1.3 .

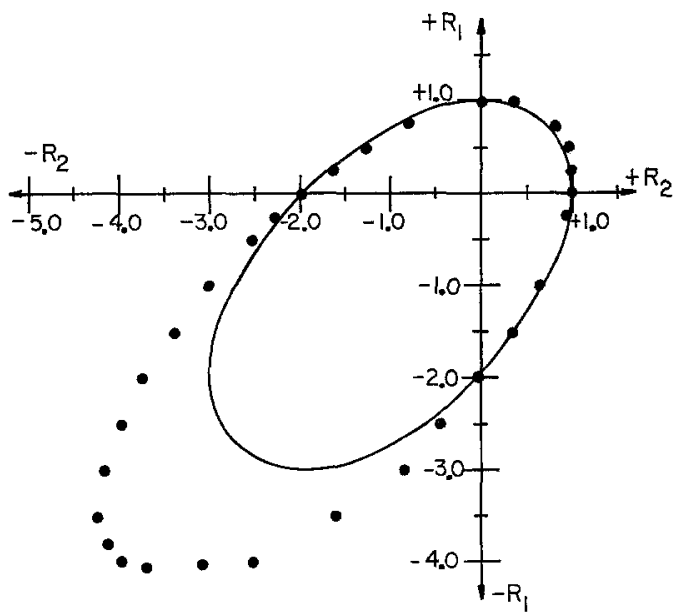

- FROM EQUATION 5 WITH $C / T=2.0$

SOLID LINE FROM EQUATION 3 WITH $C / T=2.0$

Figure 6 Comparison of results predicted by Equations 3 and 5 for a $C / T$ ratio of 2.0 .

and interest centres around the value of $\sigma$ needed to cause yielding. If these values are inserted into Equation 1, the following results:

$$
\begin{array}{r}
R=-\left(\frac{X-1}{2}\right) \pm \frac{1}{2}\left[(X+1)^{2}+\right. \\
12 B(X-1)]^{\frac{1}{2}}
\end{array}
$$

where $R=\sigma / T, X=C / T$, and $B=P / T$. In this form, the absolute value of $P$ is employed, and $C$ and $T$ are the absolute values of the compressive and tensile yield strengths obtained at atmospheric pressure.

Following a similar procedure, Equation 4 can be expressed:

$$
R=1+\frac{3 B}{2}\left(\frac{X-1}{X}\right)
$$

with the same definitions as given above.

The last two equations permit one to predict the influence of pressure upon yield strength in terms of the two yield criteria being considered. Once $X$ is determined experimentally, it is a simple matter to assign values to $B$ then compute the corresponding values of $R$. Obviously, as $P$ increases, the predicted yield strength $\sigma$ also increases. From an unpublished paper [16] sent to one of us, useful data had been consolidated from several sources [15, 17-19]. Most of those contents [16] were published subsequently [14] although the tabulated information we have used was not fully included in [14]. Figs. 7 and 8 include plots of $R$ versus $B$ computed from Equations 10 and 11; the particular value of $X$ obtained from [16] is included for each individual polymer. Experimental findings as reported elsewhere [16] are included as specific points. Since these data pertain to tensile yield stress values, the positive root of Equation 10 was used in all computations involving that equation, while Equation 11 predicts changes in tensile yield stress as a function of pressure based upon the yield criterion expressed by Equation 4 .

It may be noted that the predictions based upon Equation 11 describe a constant linear increase of yield stress with increasing pressure. Nadai [10] has pointed out that this is an unlikely phenomenon since evidence indicated a "bending back" should take place; this is just what is indicated when Equation 10 is used, as a parabolic relationship results.

There seems to be contradictory evidence on this point. The findings of Sardar et al [15] do indeed show that the change in yield stress decreases from linearity as pressure is increased; one might also infer this from the graphs in the paper by Ainbinder et al [13]. On the other hand Christiansen et al [14] show an opposite effect (see their page 459) as far as pressure is concerned.

It seems reasonable to suggest that the general behaviour of the experimental data points on 

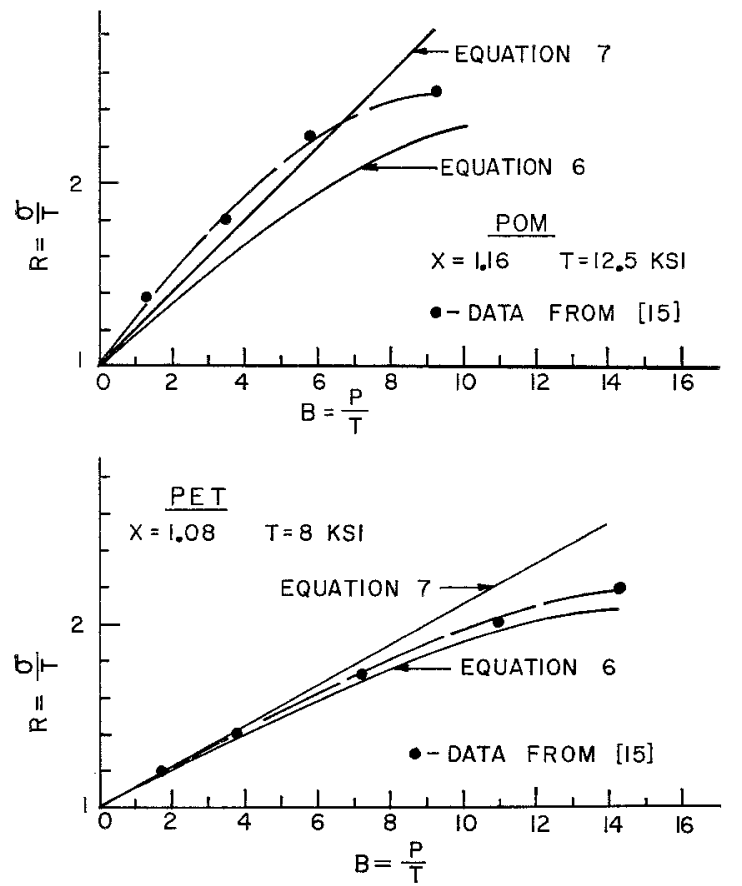

Figure 7 Effect of hydrostatic pressure on tensile yield strength of polyoxymethylene (POM) and polyethylene terephthalate (PET).
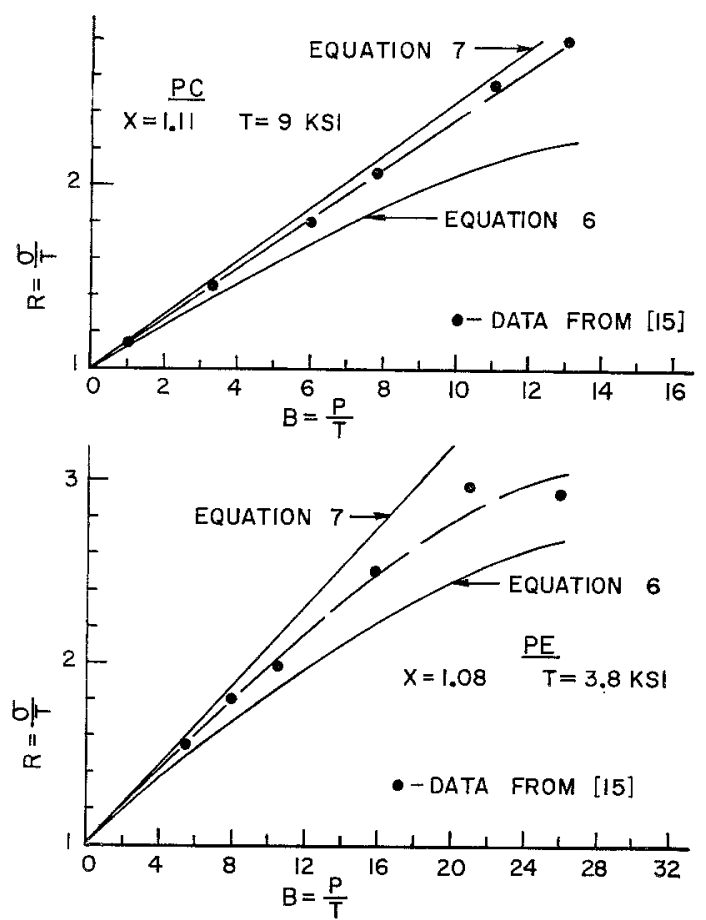

Figure 8 Effect of hydrostatic pressure on tensile yield strength of polycarbonate (PC) and polyethylene (PE).
Figs. 7 and 8 do seem to behave more like the predicted values from Equation 10 even in those instances where their absolute values are closer to the upper lines from Equation 11. Comparisons of absolute values will be affected by the values of "yield strength" selected by various investigators. As there is, apparently, no single method used to determine this property for polymers, it is not surprising that inconsistencies could arise.

\section{Conclusions}

A pressure modified von Mises yield criterion, which also includes the means for handling differences in tensile and compressive yield strengths, has been compared with experimental findings for PVC and PC. In addition, data reported by other investigators who used PMMA, PVC, and PS (polystyrene) were also utilized. In general, excellent agreement has been found. This would suggest that macroscopic yielding may be described by a single yield criterion which has, apparently, found wide acceptance when tested against a variety of materials [8]. It should be pointed out that there is little difference in the predictions of "yielding" of polymers between this criterion and the one based upon octahedral shear stress $[3,4]$ unless the ratio of compressive to tensile yield strengths is greater than unity (say $>1.5$ ). Available information in the literature would suggest that values of this ratio, at atmospheric pressure, are less than 1.5. However, under relatively high hydrostatic pressure this need not be the case. Further work must be conducted to determine whether one criterion is sounder; at this point what is needed are stress states that lead to yielding in the third quadrant of yield locus plots.

Although not fully substantiated, it appears that the criterion described by Equation 1 more properly reflects the differences in the increase of yield strength under increasing hydrostatic pressure than does the criterion based upon octahedral shear stress posed by others $[3,4]$.

\section{Acknowledgement}

Mr Raghava received some financial aid through a Union Carbide Fellowship during the early stages of this work. That assistance is gratefully acknowledged.

\section{References}

1. I. M. WARD, J. Mater. Sci. 6 (1971) 1397.

2. W. Whitney and R. D. Andrews, J. Polymer Sci. C, 16 (1967) 2981. 
3. J. C. BAUWENS, ibid A-2, 8 (1970) 893.

4. s. sternstein and L. ongchin, A.C.S. Pol. Prep. 10 (1969) 1117.

5. N. BROWN, Mat. Sci. and Eng. 8 (1971) 69.

6. A. MELDAHL, Brown Boveri Review 31 (1944) 260.

7. F. SCHLEICH ER, Z. Agnew. Math. Mech.6(1926) 199.

8. F. STASSI D'ALIA, Meccanica 3 (1967) 178.

9. N. W. tschoegl, Polymer Sci. Symp. 32 (1971) 239.

10. A. NADAI, "Theory of Flow and Fracture of Solids" (McGraw-Hill, 1950) 210, 220.

11. C. BAUWENS-CROWET, J. C. BAUWENS, and G. HOMES, J. Mater. Sci. 7 (1972) 176.

12. J. MARIN, J. H. RAUPEL, V. L. DUTTON, and M. W. Brossman, NACA Tech. Notes, No. 1536 (1948).

13. S. B. AINBINDER, M. G. LAKA, and I. YU MAIORS, Mekh. Polemerov 1 (1965) 65.

14. A. W. CHRISTIANSEN, E. BAER, and S. V. RADCliffe, Phil. Mag. 24 (1971) 451.
15. D. SARDAR, S. V. RADCLIFFE, and E. BAER, Polymer Eng. and Sci. 8 (1968) 290.

16. A. CHRISTIANSEN, S. V.RADCLIFFE, and E. BAER, communication to $\mathbf{R}$. Raghava via unpublished work, 1971.

17. K. D. PAE and D. R. MEARs, Polymer Letters 6 (1968) 269.

18. G. BIGLIONE, E. BAER, and S. V. RADCLIFFE, in "Fracture 1969" Proceeding of the second International Conference, Brighton, 1969, ed. P. L. PRATT (Chapman and Hall, London, 1969).

19. D. R. MEARS, K. D. PAE, and J. A. SAUER, $J$. Appl. Phys. 40 (1969) 4229.

Received 20 March and accepted 21 July 1972. 